\title{
Kritische Betrachtung des Referentenentwurfs zum Erneuerbaren-Energien-Gesetz 2021 im Hinblick auf die Stromerzeugung aus Photovoltaik
}

\author{
Jan Flecken
}

(c) Der/die Autor(en) 2020. Dieser Artikel ist eine Open-Access-Publikation.

\begin{abstract}
Aufgrund der rasch wechselnden Entwicklungen in der Energiebranche müssen die entsprechenden Rahmenbedingungen im EEG sowie im übrigen Recht regelmäßig justiert werden. Deshalb hat das Bundesministerium für Wirtschaft und Energie am 14. September 2020 den Gesetzesentwurf zur Novellierung des EEG veröffentlicht und den Ländern und Branchenverbänden zur Konsultation zukommen lassen. Mit diesem Gesetzesentwurf soll das geltende EEG 2017 durch ein grundlegend novelliertes EEG ersetzt werden. Die folgende Ausarbeitung beschreibt die wichtigsten Änderungen in Hinblick auf die Stromerzeugung aus Photovoltaik. Außerdem werden die geplanten Änderungen mit Hilfe von zahlreichen Stellungnahmen von Branchenvertretern kritisch betrachet und mögliche einheitliche Kritikmuster identifiziert.
\end{abstract}

\section{Einleitung}

In Deutschland ist das Erneuerbare-Energien-Gesetz (EEG) seit mehr als 20 Jahren eine zentrale Grundlage für den Ausbauerfolg der erneuerbaren Energien im Stromsektor. Seit der Einführung der ersten Fassung im Jahr 2000 stieg der Anteil erneuerbarer Energien am Stromverbrauch beständig von rund $6 \%$ im Jahr 2000 auf rund $42 \% \mathrm{im}$ Jahr 2019. Die erneuerbaren Energien entwickeln sich immer mehr von einer Nischentechnologie hin zu einem der Hauptenergieträger der deutschen Stromversorgung. Dies wird langfristig zu einer Transformation des Energieversorgungssystems führen. ${ }^{1}$

Der Erfolg des EEG hinsichtlich der Zubaumengen erneuerbarer Energien ist vor allem auf Rahmenbedingungen wie die festgeschriebene Einspeisevergütung über einen Zeitraum von zwanzig Jahren und eine zugesicherte Einspeisevergütung in Verbindung mit einer Netzanschluss- und Stromabnahmegarantie zurückzuführen. Diese Bedingungen erzeugen eine große wirtschaftliche Planungssicherheit für Anlagenbetreibende. Einhergehend mit dem stark wachsenden Anteil erneuerbarer Energien stiegen jedoch auch die Förderkosten immer weiter an. Diese werden durch die EEG-Umlage auf die Stromendverbrauchenden umgelegt. Ein wesentlicher Grund für die Kostensteigerung war die fehlende Marktintegration der erneuerbaren Energien in den deutschen Strommarkt. Wettbewerbliche Mechanismen bzw. eine der Nachfrage entsprechende Stromeinspeisung seitens der regenerativen Anlagenbetreibenden wurden durch die ersten Fassungen des EEG zunächst nicht forciert. ${ }^{2}$ Darüber hinaus führte der Ausbau der volatil einspeisenden Erneuerbaren-EnergienAnlagen zu einem erschwerten Netzmanagement. Die Befürchtung einer Reduzierung der Versorgungssicherheit kam auf. ${ }^{3}$ Auch für den liberalisierten und wettbewerblich organisierten Strommarkt stellte sich die fehlende Marktintegration der regenerativen Energien als Gefährdung dar. ${ }^{4}$

So wurde das EEG mehrfach novelliert und an die regelmäßig neu entstehenden Herausforderungen angepasst. Einen Paradigmenwechsel läutete das EEG 2017 ein. In der

Jan Flecken, M.Sc.,

RWTH Aachen University,

Aachen, Deutschland
Novelle wurde das deutsche Fördermodell von einem Einspeisevergütungssystem zu einem Ausschreibungssystem geändert. Der Gesetzgeber wollte durch diesen Systemwechsel eine kostengünstigere, sichere und marktintegrierende Förderung von erneuerbaren Energien einführen. Zunächst mit Skepsis betrachtet, entwickelte sich das Ausschreibungsmodell zu einem etablierten Mechanismus, mit dem ein kosteneffektiveres und netzfreundlicheres Voranschreiten der Energiewende gewährleistet werden konnte.

Wie bereits erwähnt, müssen aufgrund der rasch wechselnden Entwicklungen in der Energiebranche die entsprechenden Rahmenbedingungen im EEG sowie im übrigen Recht regelmäßig justiert werden. Die Klimaschutzziele müssen erreicht werden und auch die Hemmnisse im übrigen Recht, die den Ausbau der erneuerbaren Energien zum Teil bremsen, müssen beseitigt werden. Gleichzeitig kann der Ausbau der erneuerbaren Energien langfristig nur gelingen, wenn das energiewirtschaftliche Zieldreieck eingehalten wird. Hierzu gehört, dass die Kosten im Interesse einer preisgünstigen Energieversorgung und bezahlbarer Strompreise begrenzt bleiben. Mit Blick auf eine sichere und kosteneffiziente Stromversorgung müssen die erneuerbaren Energien außerdem stärker in den Strommarkt und das Stromversorgungssystem integriert werden und ihr Ausbau muss mit den dafür erforderlichen Stromnetzen synchronisiert werden. Vor dem Hintergrund dieser Herausforderungen soll das EEG nun erneut grundlegend novelliert werden und zukunftsfähige Lösungen für die beschriebenen Herausforderungen liefern. ${ }^{6}$ Dazu hat das Bundesministerium für Wirtschaft und Energie (BMWi) am 14.9. 2020 den Gesetzesentwurf zur Novellierung des EEG veröffentlicht und den Ländern und Branchenverbänden zur Konsultation zukommen lassen. Mit diesem Gesetzesentwurf soll das geltende EEG 2017 durch ein grundlegend novelliertes EEG ersetzt werden, das zum 1.1.2021 in Kraft treten soll. Stellungnahmen zum Referentenentwurf (RefEnt) konnten bis zum 17.9.2020 eingereicht werden.

Ziel dieser Ausarbeitung ist es, eine detaillierte Erläuterung der geplanten Änderungen des Gesetzesentwurfs

1) Richter, Ein Energiesystem im Wandel, Stand 23.9.2020, abrufbar unter https://www.erneuerbareenergien.de/ein-energiesystem-imwandel, S. 1.

2) Haucap, Die Marktintegration der Stromerzeugung aus Erneuerbaren Energien, 2013, S. 5.

3) Bundesministerium für Wirtschaft und Energie, Das Erneuerbare-Energien-Gesetz 2014, Stand 23.9.2020, abrufbar unter https://www.erneuerbare-energien.de/EE/Redaktion/DE/Dossier/eeg.html?cms_docId $=73930$.

4) Haucap, Die Marktintegration der Stromerzeugung aus Erneuerbaren Energien, 2013, S. 13.

5) Bundesministerium für Wirtschaft und Energie, Das Erneuerbare-Energien-Gesetz 2017, Stand 23.9.2020, abrufbar unter https://www.erneuerbare-energien.de/EE/Redaktion/DE/Dossier/eeg.html?cms_docId=401818, S. 1 .

6) Bundesministerium für Wirtschaft und Energie, Referentenentwurf - Entwurf eines Gesetzes zur Änderung des ErneuerbareEnergien-Gesetzes und weiterer energierechtlicher Vorschriften, 2020, S. 2 
Tab. 1 Im Referentenentwurf zum Erneuerbaren-Energien-Gesetz 2021 definierte jährliche Zubaumenge verschiedener Technologien für die Jahre 2021 bis $2029 .{ }^{10}$

\begin{tabular}{lllllllllll}
\hline $\begin{array}{l}\text { Bruttozubau } \\
\text { [GW] }\end{array}$ & $\mathbf{2 0 2 1}$ & $\mathbf{2 0 2 2}$ & $\mathbf{2 0 2 3}$ & $\mathbf{2 0 2 4}$ & $\mathbf{2 0 2 5}$ & $\mathbf{2 0 2 6}$ & $\mathbf{2 0 2 7}$ & $\mathbf{2 0 2 8}$ & $\mathbf{2 0 2 9}$ & $\mathbf{2 0 3 0}$ \\
\hline Wind an Land & 1,5 & 1,8 & 4,3 & 2,9 & 3,7 & 3,9 & 4,5 & 4,5 & 5,4 & Zubau außerhalb Zielmodell \\
Photovoltaik & 4,6 & 4,8 & 4,8 & 4,8 & 4,8 & 5,3 & 5,4 & 5,5 & 5,6 & Zubau außerhalb Zielmodell \\
Wind auf See & 0,5 & 0,5 & 0,7 & 0,7 & 0,7 & 0,95 & 0,95 & 0,95 & 2,9 & 3,5 plus Zubau außerhalb Zielmodell \\
Biomasse & 0,2 & 0,2 & 0,3 & 0,3 & 0,3 & 0,3 & 0,3 & 0,3 & 0,3 & 0,3 plus Zubau außerhalb Zielmodell \\
\hline
\end{tabular}

EEG 2021 im Vergleich zu seinem Vorgängergesetz EEG 2017 herauszuarbeiten. Dabei wird ein besonderer Fokus auf die Änderungen in Zusammenhang mit dem Ausbau der Photovoltaik (PV) gelegt. Es werden zunächst die relevantesten Ankerpunkte des neuen Gesetzesentwurfs erläutert, um anschließend die bedeutendsten Änderungen detailliert $\mathrm{zu}$ analysieren. Darauf folgt eine kritische Bewertung der Änderungen auf Grundlage von zahlreichen veröffentlichten Stellungnahmen verschiedener Branchenakteure. Ziel ist es, mögliche einheitliche Kritikmuster zu identifizieren.

\section{Ankerpunkte Referentenentwurf zum Erneuerbaren-Energien-Gesetz 2021}

Mit dem RefEnt soll das geltende EEG 2017 durch ein grundlegend novelliertes EEG ersetzt werden, das zum 1.1.2021 in Kraft tritt. Das EEG 2021 soll Lösungen für die drängendsten Herausforderungen liefern. Seine bedeutsamsten Inhalte gliedern sich in sechs Komplexe und werden folgend beschrieben.

\subsection{Erreichung Treibhausgasneutralität}

Im EEG 2021 soll gemäß \&1 RefEnt EEG 2021 als neue Zielsetzung verankert werden, dass der gesamte in Deutschland produzierte und verbrauchte Strom im Jahr 2050 treibhausgasneutral sein muss. Demzufolge müssen auch Stromimporte aus regenerativen Quellen stammen. Zum Erreichen dieses Ziels will sich Deutschland verstärkt für Regelungen auf europäischer Ebene einsetzen. Vor allem Stromimporte aus Frankreich sind zurzeit bedeutende Regelorgane für den deutschen Strommarkt. Aufgrund des hohen Anteils an Atomstrom im französischen Strommix sind diese Importe zumeist nicht regenerativen Ursprungs.

\subsection{Umsetzung des Klimaschutzprogramms 2030}

Im EEG soll das bereits im Klimaschutzprogramm 2030 festgelegte Ziel von 65 \% erneuerbaren Energien am Stromverbrauch im Jahr 2030 verankert werden. In der kommenden Novelle sollen die Weichen gestellt werden, um das bedeutsame Zwischenziel auf dem Weg zur Treibhausgasneutralität $2050 \mathrm{zu}$ erreichen. Dazu legt das geplante Gesetz einen genauen Ausbaupfad jeder Technologie bis zum Jahr 2030 fest.

Das Bundeswirtschaftsministerium für Wirtschaft und Energie prognostiziert einen Bruttostromverbrauch von 580 TWh im Jahr 2030. Daraus lässt sich eine angestrebte Stromerzeugung aus erneuerbaren Energien in Höhe von $377((1 / 6))$ TWh ableiten. Wegen bestehender Prognoseunsicherheiten über die tatsächliche Höhe des Bruttostromverbrauchs im Jahr 2030 wird die Voraussage gemäß \97 RefEnt EEG 2021 regelmäßig evaluiert und ggf. angepasst. ${ }^{7}$ Eine besondere Erschwernis der Prognose wird durch die gegenläufigen Entwicklungen der weiteren Anstrengungen zur Energieeffizienz einerseits und der neuen Stromverbraucher im Zuge der Sektorkopplung andererseits erzeugt.
Die im RefEnt definierten Ziele sind zum Teil noch ambitionierter ausgestaltet als die im Klimaschutzprogramm 2030, sodass eine zusätzliche Sicherheit für das Erreichen der Klimaziele geschaffen wird. ${ }^{8}$ Die Windenergie an Land soll mit einer installierten Leistung von 71 ((1/6)) GW zur Erreichung der Ziele beitragen. Im Klimaschutzprogramm 2030 wurde noch eine Spannbreite von 67 bis 71 GW definiert. Die (PV) soll im Jahr 2030 eine installierte Kapazität von $100 \mathrm{GW}$ vorweisen. Dies entspricht einer leichten Erhöhung von 2 GW im Vergleich zum Klimaschutzprogramm 2030. Außerdem wird bei der Biomasse eine Zielmarke von 8,4 GW normiert. Das Ziel der installierten Leistung von Windenergieanlagen auf See im Jahr 2030 wurde bereits mit der Novelle des Windenregie-auf-SeeGesetzes (WindSeeG) vorgelegt. ${ }^{9}$ Bei Wind auf See soll die zielerfüllungsrelevante Leistung von $20 \mathrm{GW}$ schon im ersten Quartal 2030 zur Verfügung stehen. Zusätzliche verwirklichte Anlagen in späteren Quartalen sind demnach nicht mehr relevant zur Erreichung des 65\%-Ausbauziels. Die nachfolgende Tabelle 1 zeigt die im RefEnt EEG 2021 definierten Ausbaupfade der verschiedenen Technologien für die Jahre 2021 bis 2029.

Neben der Neujustierung der Ausbaupfade der einzelnen Technologien hebt das BMWi im RefEnt die Notwendigkeit einer besseren Zusammenarbeit aller Akteurinnen und Akteure in Bund, Ländern und Kommunen hervor. Neben energierechtlichen Änderungen müssen deshalb weitere Weichen gestellt werden. Insbesondere das Planungs-, Genehmigungs- und das Natur- und Artenschutzrecht müssen die ambitionierten Ausbauziele für erneuerbare Energien widerspiegeln. Die Verfahrensdauer für die Genehmigung von Erneuerbare-Energien-Anlagen, insbesondere Windkraftanlagen, ist oft zu lang. Ein Lösungsansatz soll ein im Gesetz definierter Koordinierungsmechanismus bieten. So können die Länder die Chancen, die im Ausbau der erneuerbaren Energien liegen, für regionale Wertschöpfung und kommunale Einnahmen gezielter nutzen. ${ }^{11}$ Für den Wirtschafts-, Innovations- und Beschäftigungsstandort Deutschland sieht die Bundesregierung in entschlossenem und gemeinsamem Handeln mit den ge-

7) Bundesministerium für Wirtschaft und Energie, Referentenentwurf - Entwurf eines Gesetzes zur Änderung des ErneuerbareEnergien-Gesetzes und weiterer energierechtlicher Vorschriften, 2020, S. 83.

8) Presse- und Informationsamt der Bundesregierung, Klimaschutzprogramm 2030 der Bundesregierung zur Umsetzung des Klimaschutzplans 2050, 2020, S. 39.

9) Bundesministerium für Wirtschaft und Energie, Entwurf eines Gesetzes zur Änderung des Windenergie-auf-See-Gesetzes und anderer Vorschriften, 2020, S. 2.

10) Bundesministerium für Wirtschaft und Energie, Referentenentwurf - Entwurf eines Gesetzes zur Änderung des ErneuerbareEnergien- Gesetzes und weiterer energierechtlicher Vorschriften, 2020, S. 82.

11) Bundesministerium für Wirtschaft und Energie, Referentenentwurf - Entwurf eines Gesetzes zur Änderung des ErneuerbareEnergien- Gesetzes und weiterer energierechtlicher Vorschriften, 2020, S. 84. 
eigneten Prämissen große Chancen. Eine frühzeitige Einstellung der Unternehmen auf die Herausforderungen und Chancen für Innovation und klimafreundliches Wachstum soll dabei erfolgen. Außerdem sollen die Bürgerinnen und Bürger Möglichkeiten erhalten, sich aktiv an der Energiewende zu beteiligen. ${ }^{12}$

\subsection{Weitere Dämpfung der Kostenentwicklung}

Durch die Einführung des Ausschreibungsmodells im EEG 2017 konnten die Kosten für Neuanlagen signifikant gesenkt werden. Das Bundesministerium verdeutlicht, dass für eine hohe Akzeptanz für den Ausbau erneuerbarer Energien in der Bevölkerung eine Eindämmung der Kosten von wesentlicher Bedeutung ist. Ein Großteil der Kosten, die durch die EEG-Umlage auf die Verbraucherinnen und Verbraucher abgewälzt werden, ist auf Bestandsanlagen zurückzuführen. Vor allem PV-Anlagen benötigten zu Beginn ihrer technischen Entwicklung hohe Vergütungssätze, damit sie wirtschaftlich betrieben werden konnten. Mittlerweile sind sie die kostengünstigste regenerative Energiequelle. Für eine weitere Begrenzung der Kosten und eine Steigerung der Akzeptanz sieht das Bundesministerium folgende Maßnahmen vor.

a) Ab dem Jahr 2021 sind zur Erhöhung der Einnahmen zusätzliche Haushaltsmittel aus dem Gesamthaushalt für staatliche Zuschüsse vorgesehen. Die entsprechenden technischen Anpassungen im EEG-System wurden hierfür unlängst vorgenommen. Der zweite Nachtragshaushalt 2020 sieht hierfür die Bereitstellung von $11 \mathrm{Mrd}$. Euro vor, im Übrigen fließen ab 2021 jährlich die Mehreinnahmen aus dem Brennstoffemissionshandel zum großen Teil in das EEGFinanzierungssystem. ${ }^{13}$

b) $\mathrm{Zu}$ weiteren Reduzierung der Ausgaben enthält der Gesetzesentwurf diverse Einzelmaßnahmen. So werden die Höchstwerte in den Ausschreibungen für PV und Windenergie herabgesenkt. Eine schnellere Reaktion des atmenden Deckels soll besser auf Kostenentwicklungen der PV reagieren und eine Erweiterung der Flächenkulisse bei PV zielt auf einen noch stärkeren Wettbewerb ab.

c) Der Betrag der gesamten EEG-Umlage wurde für das Jahr 2019 auf 22,7 Mrd. Euro geschätzt. Die Wirtschaft kam dabei für 11,3 Mrd. Euro auf. Durch die unter a) beschlossenen staatlichen Zuschüsse wird die EEG-Umlage in Zukunft sinken. Eine Absenkung der EEG-Umlage könnte jedoch mittelfristig zu negativen Effekten bei der EEG-Umlagebefreiung führen. Unternehmen könnten die Schwellenwerte zur sogenannten Besonderen Ausgleichsreglung nicht mehr erreichen und ihren Anspruch darauf verlieren. Auch bei einer, im Zusammenhang mit der COVID19-Pandemie stehenden, sich fortsetzenden Rezession, könnten einige Unternehmen aus der Umlagebefreiung fallen. Damit den Unternehmen in der wirtschaftlich schwierigen Gesamtsituation keine weiteren Lasten auferlegt werden, soll die EEG-Umlagebefreiung im Gesetzesentwurf zum EEG 2021 weiterentwickelt werden. Eine Sicherheit für die Wirtschaft trotz der genannten Herausforderungen soll damit geschaffen werden. ${ }^{14}$

\subsection{Erhalt der Akzeptanz für die erneuerbaren Energien}

Eines der größten Hemmnisse beim Ausbau von Erneuerbare-Energien-Anlagen ist die oft fehlende Akzeptanz der lokalen Bevölkerung. Diesem Problem soll die Novellierung des EEG entgegenwirken. Deshalb werden im EEG 2021 gezielt Maßnahmen zur Erhöhung der Akzeptanz verankert. Bürgerinnen und Bürger sowie Standortkommunen sollen künftig an den Erträgen neuer Wind- energieanlagen beteiligt werden. Dies wurde schon im Vermittlungsausschuss zwischen Bundestag und Bundesrat vereinbart. ${ }^{15}$ Diese geplanten direkten Zahlungen sollen für eine steigende Ausweisung von Windeignungsgebieten durch die Kommunen sorgen. Mit diesem Mechanismus werden die Standortkommunen für die mit den neuen Anlagen einhergehenden Beeinträchtigungen, z. B. des Landschaftsbildes, entschädigt. Auch im Bereich der PV soll eine Akzeptanzsteigerung forciert werden. Diese soll vor allem durch Anpassungen bei dem sogenannten Mieterstrom realisiert werden. Die Bundesregierung sieht dies als bedeutenden Baustein für die Akzeptanz der Energiewende, da dadurch eine direkte Partizipation von Mieterinnen und Mietern an der Energiewende ermöglicht wird.

\subsection{Stärkung der Netz- und Marktintegration}

Auch für die Stärkung der Netz- und Marktintegration enthält der Gesetzesentwurf ein Bündel an Einzelmaßnahmen. Es soll eine sogenannte Südquote eingeführt werden. Dadurch soll die regionale Steuerung verbessert und eine Reduzierung der Systemkosten erreicht werden. Bei den Ausschreibungen für Windenergieanlagen an Land soll eine Quote in Höhe von 5\% in den Jahren 2021 bis 2023 und von $20 \%$ ab dem Jahr 2024 eingeführt werden. Ebenfalls soll eine Quote von $50 \%$ für Biomasseanlagen ab dem Jahr 2024 verankert werden. Die Maßnahmen sollen sich entlastend auf den Netzengpass in der Mitte Deutschlands auswirken und eine flexiblere Stromerzeugung in Süddeutschland fördern. Dazu wird das zurzeit bestehende Netzausbaugebiet aufgehoben.

Eine weitere maßgebliche Änderung zur Verbesserung der Marktintegration der erneuerbaren Energien ist eine Weiterentwicklung der Marktprämie. Neu errichtete Erneuerbare-Energien-Anlagen erhalten gemäß Gesetzesentwurf keine Vergütung bei gewissen zeitlichen Abfolgen von negativen Strombörsenpreisen. Durch diese Maßnahme werden zugleich Anreize für Speichertechnologien und neue Perspektiven für Innovationen gesetzt. Des Weiteren sollen die Anforderungen an die Steuerbarkeit von Erneuerbare-Energien-Anlagen ausgeweitet und die Digitalisierung soll durch sogenannte Smart-Meter-Gateways fortgeführt werden. ${ }^{16}$

\subsection{Einstieg in die „Post-Förderung- ̈̈ra“}

Im Jahr 2021 läuft der 20-jährige Vergütungszeitraum der ersten durch das EEG geförderten Anlagen aus. Deshalb soll in der Gesetzesnovelle der Rechtsrahmen für diese Anlagen angepasst werden. Der Anspruch auf vorrangige Einspeisung bleibt bereits nach jetziger Rechtslage bestehen. Anlagenbetreibende können ihren Strom direkt vermarkten und dadurch Markterlöse für den Weiterbetrieb erzielen. Der Weiterbetrieb kleiner Anlagen in der Di-

12) Presse- und Informationsamt der Bundesregierung, Klimaschutzprogramm 2030 der Bundesregierung zur Umsetzung des Klimaschutzplans 2050, 2020.

13) Bundesministerium für Wirtschaft und Energie, Referentenentwurf - Entwurf eines Gesetzes zur Änderung des ErneuerbareEnergien-Gesetzes und weiterer energierechtlicher Vorschriften, 2020, S. 82.

14) Bundesministerium für Wirtschaft und Energie, Referentenentwurf - Entwurf eines Gesetzes zur Änderung des ErneuerbareEnergien-Gesetzes und weiterer energierechtlicher Vorschriften, 2020 , S. 84.

15) Bundesrat, Vermittlungsausschuss erzielt Kompromiss zum Klimapaket, Stand 23.9.2020, abrufbar unter https://www.bundesrat.de/SharedDocs/pm/2019/015.html, S. 1.

16) Bundesministerium für Wirtschaft und Energie, Referentenentwurf - Entwurf eines Gesetzes zur Änderung des ErneuerbareEnergien-Gesetzes und weiterer energierechtlicher Vorschriften, 2020, S. 84. 
rektvermarktung ist zurzeit als eher unwirtschaftlich einzuschätzen. Aufgrund dessen wird übergangsweise bis zu ihrer vollständigen Marktintegration eine Alternative zur Direktvermarktung im Gesetzesentwurf geboten. Laut RefEnt EEG 2021 können die Anlagenbetreibenden den in der Anlage erzeugten Strom bis Ende 2027 dem Netzbetreiber zur Verfügung stellen und erhalten hierfür den Marktwert abzüglich der Vermarktungskosten. Der Gesetzgeber will dadurch den Abbau dieser Anlagen und ein „,unkontrolliertes Einspeisen“"verhindern. ${ }^{17}$

\section{3. Änderung des Erneuerbare-Energien-Gesetzes}

Im Folgenden wird auf die relevantesten Änderungen des EEG eingegangen, mit denen das BMWi anstrebt, die zuvor dargestellten Ankerpunkte zu verwirklichen. Eine Schwerpunktauslegung wird dabei vor allem auf Änderungen, die den Ausbau der PV betreffen, gelegt. Bei den behandelten Änderungen wird zu Beginn auf technologieübergreifende Abwandlungen eingegangen, die zum Teil auch den Ausbau der PV betreffen. Anschließend werden die Änderungen mit direktem Bezug auf die photovoltaische Stromerzeugung analysiert. Die Vorgehensweise bei der Analyse besteht darin, dass zuerst die geplante Gesetzesänderung neutral erläutert und darauffolgend eine kritische Bewertung erarbeitet wird. Bei der kritischen Betrachtung wird vor allem auf die zahlreichen Stellungnahmen der Branchenvertretenden Bezug genommen und ein Konsens der häufigsten Kritikpunkte dargestellt.

\subsection{Technologieübergreifende Änderungen \\ 3.1.1 Zu $\$ 1$ Zweck und Ziel des Gesetzes}

Unter $₫ 1$ Abs. 3 RefEnt EEG 2021 wird das bisherige Ziel, einen Anteil erneuerbarer Energien von mindestens $80 \%$ am Bruttostromverbrauch bis zum Jahr 2050 zu erreichen, aktualisiert. Der vorliegende Entwurf sieht vor, dass im Jahr 2050 der gesamte Strom, der im Staatsgebiet der Bundesrepublik Deutschland erzeugt oder verbraucht wird, treibhausgasneutral erzeugt wird. ${ }^{18}$ Zudem soll der erforderliche Ausbau gemäß $₫ 1$ Abs. 4 RefEnt EEG 2021 stetig, kosteneffizient und netzverträglich erfolgen.

Kritische Betrachtung: Die Festschreibung des Ziels der Treibhausgasneutralität vor dem Jahr 2050 im EEG wird von einer Vielzahl der Branchenakteurinnen und -akteure begrüßt. Das Zwischenziel eines $65 \%$ igen Anteils der erneuerbaren Energien bezieht sich jedoch nach Meinung vieler Akteurinnen und Akteure auf einen zu geringen Wert von 580 ((1/6)) TWh. Mit der im Bundes-Klimaschutzgesetz bereits festgeschriebenen Treibhausgasminderung in allen Energiesektoren, einer beschlossenen Wasserstoffstrategie und einer deutlich erkennbaren Umstellung der Pkw-Flotte auf Elektroantriebe in diesem Jahrzehnt ist die Annahme für den Bruttostromverbrauch unrealistisch. ${ }^{19}$ Dass die im vorliegenden Entwurf getroffenen Vermutungen zum Stromverbrauch nicht zutreffen, zeigen auch die Ansichten einer Vielzahl von Marktforschenden und Fachleuten, die einen steigenden Stromverbrauch im Jahr 2030 voraussagen. Der Bundesverband Erneuerbare Energien (BEE) prognostiziert einen Bruttostromverbrauch im Jahr 2030 von mindestens 740 ((1/6)) TWh. Demnach müssten 481 ((1/6)) TWh Ökostrom im Jahr 2030 produziert werden, um das $65 \%$-Ziel zu erreichen. Ähnliche Prognosen in dieser Größenordnung finden sich in der Leitstudie 2018 der Deutschen Energie-Agentur (dena), der Analyse des Energiewirtschaftlichen Instituts an der Universität zu Köln (EWI) und einer Studie des Bundesministeriums für Verkehr und digitale Infrastruktur (BMVi). ${ }^{20}$

3.1.2 Zu \51 Verringerung des Zahlungsanspruchs bei negativen Preisen

Nach $₫ 51$ Abs. 1 RefEnt EEG 2021 verringert sich der anzulegende Wert auf null für den gesamten Zeitraum, in dem der Spotmarktpreis, wie in $\$ 3 \mathrm{Nr}$. 42a definiert, in mindestens 15 aufeinanderfolgenden Minuten negativ ist. Damit würde die bestehende sogenannte 6-Stunden-Regel nach $\$ 51$ Abs. 1 EEG 2017 in der Praxis zu einer 15-Minuten-Regel gekürzt. ${ }^{21}$ Der Wegfall der Förderung bei negativen Preisen gilt ausschließlich für Neuanlagen. Bei Bestandsanlagen entfällt die Förderung für negative Preise weiterhin erst nach mindestens sechs aufeinanderfolgenden Stunden negativer Spotmarktpreise. ${ }^{22}$ Hier gilt insofern Vertrauensschutz.

Kritische Betrachtung: Der Entfall des Anspruchs auf eine Marktprämie im Fall negativer Preise ist laut Aussage einiger Branchenvertreter mit einer Marktprämienkürzung zu vergleichen. Eine Flexibilisierung des Stromsystems wird durch diese Änderung nicht erreicht. Im Gegenteil wird sogar durch die frühere Abschaltung von ErneuerbareEnergien-Anlagen der Flexibilisierungsdruck von konventionellen Erzeugern genommen. ${ }^{23}$ Eine Verschärfung der Verringerung des Zahlungsanspruchs bei negativen Preisen lehnen deshalb die meisten Branchenvertretenden entschieden ab. Die geplante Änderung legt offen, dass der Gesetzgeber nicht im Sinne eines erneuerbaren Stromsystems denkt. Es braucht ein neues Strommarktdesign und die gleichzeitige Abschaffung von $\$ 51$. Die Verringerung des Zahlungsanspruchs in Stunden mit negativen Marktpreisen wird die Finanzierbarkeit der Anlagen erheblich erschweren. Die Investorin oder der Investor muss vor dem Kauf und der Inbetriebnahme der Anlage die Rückzahlung des Kredites planen können. Der Entfall der Vergütung bei negativen Spotmarktpreisen für bereits 15 Minuten verursacht eine Planungsunsicherheit, die dazu führt, dass die anfallenden Zinsen für die Finanzierung steigen und damit letztendlich auch die Kosten für die Endverbrauchenden. Es werden jedoch nicht nur die Projekte teurer, die Vergütungen werden, wie vom Gesetzgeber gewünscht, durch solche Regelungen auch nicht sinken. Damit werden die Kosten für die Verbraucherinnen und Verbraucher zusätzlich gesteigert. Diese Problematik verschärft sich durch den aktuell zu beobachtenden deutlichen Anstieg der Stunden mit negativen Marktpreisen. So gab es bisher im Jahr 2020 mit 166 Stunden bereits mehr negative Stunden an der Strombörse als im gesamten Jahr 2019 (122 Stunden). Zahlreiche Energieexpertinnen und -experten gehen davon aus, dass diese Tendenz - unabhängig von der Corona-Pandemie in den kommenden Jahren steigen wird. ${ }^{24}$

17) Bundesministerium für Wirtschaft und Energie, Referentenentwurf - Entwurf eines Gesetzes zur Änderung des ErneuerbareEnergien-Gesetzes und weiterer energierechtlicher Vorschriften, 2020, S. 84.

18) Bundesverband Erneuerbare Energie, Stellungnahme des Bundesverbands Erneuerbare Energie zum Entwurf eines Gesetzes zur Änderung des Erneuerbare-Energien-Gesetzes, 2020, S. 5.

19) Bundesverband Neue Energiewirtschaft, Stellungnahme zum Entwurf eines Gesetzes zur Änderung des Erneuerbare-Energien-Gesetzes, 2020, S. 7.

20) Bundesverband Erneuerbare Energie, Stellungnahme des Bundesverbands Erneuerbare Energie zum Entwurf eines Gesetzes zur Änderung des Erneuerbare-Energien-Gesetzes, 2020.

21) Bundesverband WindEnergie, Stellungnahme zum Entwurf eines Gesetzes zur Änderung des EEG und weiterer energierechtlicher Vorschriften, 2020, S. 37.

22) Bundesministerium für Wirtschaft und Energie, Referentenentwurf - Entwurf eines Gesetzes zur Änderung des ErneuerbareEnergien-Gesetzes und weiterer energierechtlicher Vorschriften, 2020, S. 133

23) Bundesverband Neue Energiewirtschaft, Stellungnahme zum Entwurf eines Gesetzes zur Änderung des Erneuerbaren-Energien-Gesetzes und weiterer energierechtlicher Vorschriften, 2020, S. 4.

24) Bundesverband der Energie- und Wasserwirtschaft, Stellungnahme zum Referentenentwurf für ein Gesetz zur Änderung des EEG, 2020, S. 9. 
Eine Vielzahl an Branchenverbänden fordert, dass die Regelung in $\$ 51$ zur Verringerung des Zahlungsanspruchs bei negativen Preisen gestrichen wird und stattdessen geeignete Anreizmechanismen für die Bereitstellung von Flexibilitäten eingeführt werden. Eine Möglichkeit ist beispielsweise die Anpassung der Umlagen- und Abgabenregelungen für Anlagen, die systemdienliche Flexibilitäten bereitstellen können. Eine weitere Möglichkeit ist die Einführung eines regionalen Flexibilitätsmarktes. ${ }^{25}$

\subsubsection{Zu \9 Digitalisierung der Energiewende}

Der RefEnt gestaltet den $\$ 9$ EEG neu. Hiernach müssen die Betreibenden von Anlagen mit einer installierten Leistung von mehr als $1 \mathrm{~kW}$, die in Betrieb genommen wurden, nachdem das Bundesamt für Sicherheit in der Informationstechnik bekanntgegeben hat, dass die technische Möglichkeit für die Ausstattung mit einem intelligenten Messsystem nach dem Messstellenbetriebsgesetz für die entsprechende Einbaugruppe besteht, ihre Anlagen mit technischen Einrichtungen ausstatten, mit denen die oder der Netzbetreibende oder eine andere berechtigte Person jederzeit über ein intelligentes Messsystem die Ist-Einspeisung abrufen und die Einspeiseleistung stufenweise odersobald die technische Möglichkeit besteht - stufenlos ferngesteuert regeln kann. ${ }^{26}$

Kritische Betrachtung: Eine weiter verbesserte Digitalisierung im Energiesektor ist sinnvoll. Lösungen müssen aber auch den Anforderungen der Kundinnen und Kunden sowie der Marktakteurinnen und -akteure genügen.

Den Pflichteinbau intelligenter Messsysteme (iMSys) bei Erneuerbare-Energien-Anlagen ab einer Leistung von $1 \mathrm{~kW}$ vorzusehen, ist nach Meinung vieler Branchenvertretender überzogen. Die zusätzlich erforderliche Messtechnik darf die Erlöse der Anlagen nicht wieder aufzehren. Messtechnische Anforderungen sollten dringend auf ihre Notwendigkeit überprüft werden. ${ }^{27}$

In $\$ 9$ Abs. 2 Ziff. 2b EEG 2017 war eine Spitzenlastkappung auf $70 \%$ für Anlagen bis $30 \mathrm{kWp}$ vorgesehen. Dieser Punkt ist im RefEnt EEG 2021 nicht mehr enthalten. Um Netzüberlastungen zu vermeiden, hatten insbesondere Anlagenbetreibende kleiner PV-Anlagen bisher die Möglichkeit, die Einspeiseleistung ihrer Anlage auf $70 \%$ zu reduzieren. Diese effektive und kostengünstige Lösung für Anlagen bis $30 \mathrm{kWp}$ hatte den Einbau kostspieliger Steuerungstechnik bisher überflüssig gemacht und den netzverträglichen Betrieb von kleinen PV-Anlagen ermöglicht. Die Streichung dieser Regelung kann nun für die Anlagenbetreibenden große finanzielle Aufwendungen zur Folge haben. Zudem fällt auch bei den Netzbetreibenden ein erhöhter Verwaltungsaufwand an. Einige Branchenvertretende, darunter der Bundesverband Neue Energiewirtschaft e.V., plädieren daher dafür, dass die 70\%-Regel im Sinne des wirtschaftlichen Betriebs kleinerer Anlagen beibehalten wird. ${ }^{28}$

\subsection{Photovoltaik betreffende Änderungen}

\subsection{1 $\mathrm{Zu} \llbracket 48$ a Mieterstrom}

Der vorliegende RefEnt ersetzt mit der Einführung des \48a EEG 2021 und der Aufhebung des $\$ 23$ b Abs. 1 EEG 2017 den bestehenden Mieterstromzuschlag durch einen neu anzulegenden Wert. Dieser Wert beträgt für Anlagen

- bis $10 \mathrm{kWp:} 2,66 \mathrm{ct} / \mathrm{kWh}$

- bis $40 \mathrm{kWp}: 2,40 \mathrm{ct} / \mathrm{kWh}$

- bis $750 \mathrm{kWp:} 1,42 \mathrm{ct} / \mathrm{kWh}$.

Kritische Betrachtung: Die meisten Branchenverbände begrüßen die Einführung eines gestaffelten Mieterstromzuschlags gemäß \48a EEG 2021. Allerdings sollte der Mieterstromzuschlag bei 3,5 bis $4 \mathrm{ct} / \mathrm{kWh}$ liegen. ${ }^{29}$ Mit dieser Maßnahme kann der administrative Aufwand für eine begrenzte Zeit ausgeglichen werden.

Das aktuell gültige Mieterstrom-Modell hat nach Meinung vieler Experten und Expertinnen erhebliche Schwä- chen. Wesentliche Gründe für den Zubau um nur insgesamt $18 \mathrm{MW}$ mit Mieterstromförderung bis Mitte 2020 liegen vor allem darin, dass die Kosten für das Geschäftsmodell an sich und insbesondere für dessen Abwicklung nicht mehr durch den Mieterstromzuschlag aufgefangen werden können. So haben Vermieter oder Anbieter in der Rolle als Stromlieferant einen Aufwand für die Akquise der Mieterinnen und Mieter zu leisten und müssen zudem die Stromlieferverträge mit den Mietparteien verwalten. Hinzu kommen aufwendige Messsysteme. Die Kosten für den Messstellenbetrieb werden auf jährlich rund 100 bis $150 €$ pro Teilnehmer und die Investition in die Umsetzung des Messkonzepts werden auf ebenfalls 100 bis $150 €$ pro Teilnehmer geschätzt. ${ }^{30}$ In der Anfangsphase des Mieterstrommodells konnten die die administrativen Kosten über den Mieterstromzuschlag gedeckt werden. Aufgrund starker Degression sank auch der von den anzulegenden Werten für Dach-PV-Anlagen abhängige Mieterstromzuschlag für neue Mieterstromprojekte mittlerer Größe schließlich auf null. Die Höhe der Mieterstromförderung muss deshalb auf ein attraktives und langfristig verlässliches Niveau angehoben werden, damit dieses Geschäftsmodell eine deutliche Wiederbelebung erfährt und einen signifikanten Beitrag zum Klimaschutz leistet. Hierfür dürften die vorgeschlagenen Mieterstromzuschläge von 2,66 bis 1,42 ct/kWh laut Meinung einiger Experten je nach Leistungsklasse nicht ausreichend sein. ${ }^{31}$

Befürwortet wird die Änderungsmaßnahme, dass für die Ermittlung der Leistungsgrenzen für die Förderhöhe die Zusammenfassung von Anlagen im neuen \$48a EEG-ReEnt nicht erfolgen sollte, wenn sie nicht denselben Verknüpfungspunkt haben. Nicht nachvollziehbar ist dagegen, dass eine Nicht-Zusammenfassung auf den Fall beschränkt sein soll, dass es sich um verschiedene Anlagenbetreiber handelt. Diese Voraussetzung wird in der Begründung des Gesetzesentwurfs nur beschrieben, aber nicht begründet. Diese Regelung schließt in der Realität nahezu alle Mieterstromprojekte aus. Zwar sind theoretische Konstellationen vorstellbar, in denen zwei Mieterstromprojekte in direkter Nachbarschaft innerhalb von 12 Monaten in Betrieb gehen, für die diese Lösung hilfreich wäre. In der Praxis wird die Anlagenzusammenfassung jedoch in nahezu allen Fällen in zusammenhängenden Siedlungsstrukturen angewandt. Solche Siedlungsstrukturen sind historisch bedingt meist in dem Eigentum von einer Person, die entweder selbst Betreiberin oder Betreiber wird oder zur Betreibung einen Dienstleister beauftragt. Die Einschränkung auf ,verschiedene Anlagenbetreiber" hat vermutlich das Ziel, ein künstliches Anlagensplitting durch eine betreibende Person zu verhindern. Dies ist jedoch nicht praktikabel. Aufgrund der wesentlichen Kosten für Messtechnik pro Anschlusspunkt (typischerweise ca. 30\% der Projektkosten)

25) Landesverband Erneuerbare Energien NRW, Stellungnahme Referentenentwurf EEG 2021, 2020, S. 18-21.

26) Bundesministerium für Wirtschaft und Energie, Referentenentwurf - Entwurf eines Gesetzes zur Änderung des ErneuerbareEnergien-Gesetzes und weiterer energierechtlicher Vorschriften, 2020, S. 5.

27) Bundesverband Neue Energiewirtschaft, Stellungnahme zum Entwurf eines Gesetzes zur Änderung des Erneuerbare-Energien-Gesetzes, 2020, S. 4.

28) Landesverband Erneuerbare Energien NRW, Stellungnahme Referentenentwurf EEG 2021, 2020, S. 4.

29) Bundesverband der Energie- und Wasserwirtschaft, Stellungnahme zum Referentenentwurf für ein Gesetz zur Änderung des EEG, 2020, S. 9

30) Bundesministerium für Wirtschaft und Energie, Mieterstrombericht nach $\$ 99$ des Erneuerbare-Energien-Gesetzes 2019, 2020.

31) Bundesverband der Energie- und Wasserwirtschaft, Stellungnahme zum Referentenentwurf für ein Gesetz zur Änderung des EEG, 2020, S. 36. 
wird auch eine einzelne Betreiberin oder ein einzelner Betreiber kein technisch nicht erforderliches Anlagensplitting vornehmen, sobald sie oder er es vermeiden kann. Daher schlagen viele Branchenvertretende vor, die Formulierung ,verschiedenen Betreibern' zu streichen. ${ }^{32}$

\subsubsection{Zu $₫ 22$ Ausschreibungen für Photovoltaik-}

Dachanlagen

Der RefEnt sieht unter $\$ 22$ Abs. 3 Satz 2 die Einführung eines Ausschreibungssystems für Gebäude-PV-Anlagen vor. Die Ausschreibungsgrenze soll dabei von heute 750 $\mathrm{kWp}$ schrittweise gesenkt werden. Zuerst auf $500 \mathrm{kWp}$ für die Jahre 2021 und 2022, dann auf $300 \mathrm{kWp}$ ab 2023 und schließlich auf $100 \mathrm{kWp}$ für Anlagen, die ab 2025 in Betrieb genommen werden. Die Möglichkeit, auch außerhalb von Auktionen eine Förderberechtigung zu erlangen, reduziert sich gleichzeitig von 2,3 auf 1,3 GW im Jahr 2028. ${ }^{33}$

Kritische Betrachtung: Eine Vielzahl der Marktakteure sehen eine solche Absenkung der Ausschreibungsgrenze aus mehreren Gründen sehr kritisch. Sie würde eines der wichtigsten PV-Marktsegmente weitgehend ausbremsen. Nicht nachvollziehbar erscheint in diesem Zusammenhang auch, dass mit der im Entwurf vorgesehenen Einführung eines Ausschreibungssystems für Dachanlagen gleichzeitig der Eigenverbrauch von Solarstrom stärker reguliert werden soll. Der überwiegende Teil der heute installierten Dachanlagen wird jedoch genau mit der Intention gebaut, den eigenerzeugten Solarstrom auch anteilig zu nutzen und damit an der Energiewende aktiv teilnehmen zu können. Das Marktsegment der Gebäude-PV unterscheidet sich grundlegend von dem Marktsegment der Freiflächen-PV, unter anderem durch eine höhere Komplexität, deutlich längere Vorlaufzeiten im Planungsprozess und eine gänzlich andere Investorenstruktur mit einer deutlich geringeren Risikobereitschaft und erheblichen Finanzierungsproblemen. ${ }^{34}$

Folgenden Gründe sprechen aus Sicht einiger Branchenvertreter grundsätzlich gegen die Einführung von Ausschreibungen bei PV-Anlagen auf oder an Gebäuden:

- In der Regel ist ein Investor für PV-Dachanlagen ein kleines oder mittleres Unternehmen. Eine Beteiligung an einer Ausschreibung wird zumeist gemieden, da sie zusätzliche Risiken und Vorlaufkosten im Zusammenhang mit einer PV-Investition scheuen.

- Der bürokratische Aufwand, der mit Ausschreibungen einhergeht, wäre unverhältnismäßig hoch. Dies trifft insbesondere für gewerbliche Investoren und Mittelständler zu, welche zumeist nur einmal eine solche Investition tätigen. Der bürokratische Aufwand steht in keinem vernünftigen Verhältnis zur erwarteten Einsparung bzw. Rendite.

- Im Marktsegment der PV-Dachanlagen sind durch unterschiedliche Randbedingungen, z. B. im Hinblick auf Stromtarife, Nutzerverhalten und bauliche Beschaffenheit, die Investitionen stark unterschiedlich. Faire Wettbewerbsbedingungen lassen sich hier mit vertretbarem Aufwand im Rahmen eines Auktionsverfahrens nicht schaffen

- Bei Sanierung, Bau und Planung größerer Gebäude spielen PV-Dachanlagen heute bereits in der frühen Bauphase eine wichtige Rolle. Die Energiekonzepte der Gebäude sind schon zu Beginn der Planungs- und Bauphase fest beschlossen. Die Unsicherheit einer Ausschreibung wäre eine massive Planungsunsicherheit. Sie dürfte die notwendige Berücksichtigung der PV bereits in einem frühen und kleinen Zeitfenster des Planungsprozesses zumeist verhindern und damit unmöglich machen oder andernfalls zu erheblichen Ineffizienzen und Mehrkosten führen.

Aus den genannten Gründen empfehlen verschiedene Brachenvertreter von einer schrittweisen Absenkung der Ausschreibungsgrenze auf $100 \mathrm{kWp}$ abzusehen. Stattdessen wird eine Anhebung der Ausschreibungsgrenze auf $1 \mathrm{MW}$ angeregt. ${ }^{35}$

Hinzu komm noch, dass gemäß \$38e RefEnt EEG 2021 eine Sicherheit bei den Ausschreibungen für Solaranlagen auf Gebäuden zu stellen ist. Es muss eine Sicherheit von $70 €$ pro Kilowatt Gebotsmenge bereitgestellt werden. Die Höhe der Sicherheit führt zu einer zusätzlichen wirtschaftlichen Belastung für den Anlagenbetreiber. Es ist nicht nachvollziehbar, warum eine höhere Sicherheit gefordert wird als bei der PV-Freiflächenanlagen nach $\ 55$ Abs. 2 RefEnt EEG 2021. ${ }^{36}$

\subsubsection{Zu $₫ 37$ Erweiterung der Flächenkulisse}

Gemäß §37 Abs. 1 Ziff. 2c RefEnt EEG 2021 ist eine Erweiterung der Flächenkulisse für PV-Freiflächenanlagen vorgesehen. Demnach soll die nutzbare Fläche der Randstreifen entlang von Autobahnen und Schienenwegen von 110 Meter auf 220 Meter erhöht werden. Weiter soll nach $\$ 37$ Abs. 3 RefEnt EEG 2021 die maximale Größe von PV-Freiflächenprojekten in den Ausschreibungen von derzeit zehn auf $20 \mathrm{MW}$ steigen.

Kritische Betrachtung: Die erhöhte Gebotsgröße von $20 \mathrm{MW}$ und ausgeweitete Flächenkulisse an Verkehrswegen auf 220 Meter wird von den meisten Branchenvertretern als positiv gewertet. Jedoch sollte die Regelung flexibler in Bezug auf die realen Flurstücke ausgelegt werden. Diese Maßnahme steigert die Kosteneffizienz, da die vollständige Nutzung zu potenziell günstigeren Pachtpreisen pro Fläche führt. Hintergrund ist, dass Flurstücke selten parallel zu Schienenwegen und Autobahnen angeordnet sind und so oft nur teilweise im bebaubaren Korridor liegen. ${ }^{37}$

\subsubsection{Zu $\ 49$ Degressionsmechanismus}

Der Degressionsmechanismus gemäß $₫ 49$ RefEnt EEG 2021 verfolgt die Zielsetzung, mittels einer Nachjustierung der EEG-Vergütungssätze/Marktprämien die Ausbaumengen von PV-Anlagen mit festen Vergütungssätzen (gemäß $\$ 48$ EEG) an den Ausbauzielen der Bundesregierung auszurichten. ${ }^{38}$

Kritische Betrachtung: Der Degressionsmechanismus wird im Grundsatz von allen Branchenverbänden als ein geeignetes Instrument der Mengensteuerung anerkannt.

Die im RefEnt vorgesehene geringfügige Anhebung des PV-Ausbaupfads für PV-Anlagen bis $750 \mathrm{kWp}$ von 1.900 auf 2.300 MW ist in Anbetracht der notwendigen Ausbaumenge zum Erreichen der Klimaziele, wie bereits ausgeführt, deutlich zu niedrig. Das führt gegenwärtig dazu, dass die anzulegenden Werte zur Ermittlung der EEG-Marktprämien weiterhin stark sinken. Dadurch wird der jährliche PV-Zubau von Anlagen bis $750 \mathrm{kWp}$ von ca. $3 \mathrm{GW}$ im vergangenen Jahr absehbar deutlich abnehmen und sich

32) Bundesverband der Energie- und Wasserwirtschaft, Stellungnahme zum Referentenentwurf für ein Gesetz zur Änderung des EEG, 2020, S. 35.

33) Bundesverband Erneuerbare Energie, Stellungnahme des Bundesverband Erneuerbare Energie zum Entwurf eines Gesetzes zur Änderung des Erneuerbare-Energien-Gesetzes, 2020, S. 23.

34) Bundesverband Erneuerbare Energie, Stellungnahme des Bundesverbands Erneuerbare Energie zum Entwurf eines Gesetzes zur Änderung des Erneuerbare-Energien-Gesetzes, 2020, S. 24.

35) Bundesverband Erneuerbare Energie, Stellungnahme des Bundesverbands Erneuerbare Energie zum Entwurf eines Gesetzes zur Änderung des Erneuerbare-Energien-Gesetzes, 2020, S. 25.

36) Bundesverband Neue Energiewirtschaft, Stellungnahme zum Entwurf eines Gesetzes zur Änderung des Erneuerbare-Energien-Gesetzes, 2020, S. 14.

37) Bundesverband der Energie- und Wasserwirtschaft, Stellungnahme zum Referentenentwurf für ein Gesetz zur Änderung des EEG, 2020, S. 67.

38) Bundesverband Solarwirtschaft, Stellungnahme EEG-Novelle 2021, 2020, S. 7 
bei ca. 2,3 GW im Jahr einpendeln. Zwar werden auch die Preise von Solarmodulen absehbar weiter sinken, gleichzeitig sind aber zuletzt die Nebenkosten, etwa für Zähler, stark gestiegen. ${ }^{39}$

\subsubsection{Zu $\$ 21$ und $\$ 23$ Post-EEG-Regelung}

Der Entwurf sieht vor, Anlagen bis höchstens $100 \mathrm{~kW}$ eine zweite Anschlussperspektive zu bieten. Dabei soll eine auf sie zugeschnittenen Einspeisevergütung gezahlt werden. Zudem wird für Betreiber ausgeförderter Anlagen gewährleistet, dass der Netzbetreiber die Abnahme und Vermarktung des erzeugten Stroms übernimmt. Damit bleibt der Einspeisevorrang bestehen. Diesen Anlagen wird eine Anschlussförderung in Höhe des Marktwerts abzüglich einer Vermarktungspauschale von $0,4 \mathrm{ct} / \mathrm{kWh}$ gewährt. ${ }^{40}$

Kritische Betrachtung: Die Abnahme des Stroms wird durch diese Regelung weiterhin gewährleistet. Damit ist im Ansatz eine Auffangregelung geschaffen, welche von den meisten Branchenverbänden unterstützt wird. Jedoch ist diese Regelung bis zum Jahr 2027 befristet und kann nur in Anspruch genommen werden, wenn der Strom entweder vollständig eingespeist wird oder wenn die Eigenverbrauchsanlage über ein intelligentes Messsystem nach $\$ 21$ Abs. 2 RefEnt EEG 2021 verfügt. Aus Sicht vieler Branchenvertreter ist es kritisch zu sehen, dass Anlagenbetreiber, die auf Eigenversorgung umstellen wollen, diese Anschlussregelung für ihren überschüssigen Strom nur dann nutzen dürfen, wenn sie ein mit erheblichen Zusatzkosten verbundenes intelligentes Messsystem verbauen. Dies betrifft vor allem PV-Kleinstanlagen. In der vorliegenden Fassung ist der Weiterbetrieb so formuliert, dass Eigenverbrauch und Energiemanagement im bzw. am Gebäude, auch in Verknüpfung mit $\$ 9$ Abs. 1a RefEnt EEG 21, diskriminiert wird. Eine dezentrale und verbrauchsnahe Stromerzeugung, beispielsweise auch in Verknüpfung mit einem Speicher, muss jedoch als ein wesentlicher Treiber der Energiewende angesehen werden. Dadurch wird eine Partizipation der Bevölkerung initiiert und sichert eine erhöhte öffentliche Akzeptanz. ${ }^{41}$ Ein Weiterbetrieb ohne geplanten Eigenverbrauch ist bei kleinen Anlagen bis $5 \mathrm{~kW}$ unwirtschaftlich. Die volle Weitereinspeisung mit einer Vergütung in Höhe des Marktwerts abzüglich Vermarktungskosten von $0,4 \mathrm{ct} / \mathrm{kWh}$ ergibt nach Schätzungen für 2021 eine Betreibervergütung von 2,1 ct/kWh. Diese Vergütung ist nach Meinung vieler Branchenvertreter nicht genug, um allein die jährliche Versicherung, Zählerkosten, Wartung und eventuelle Kleinreparaturen zu bezahlen. Ein Weiterbetrieb der aktuell am meisten betroffenen Anlagen im Bereich zwischen 1 und $5 \mathrm{kWp}$ ist mit dieser Regelung wirtschaftlich nicht möglich. ${ }^{42}$

\subsubsection{Ausbaupfad Photovoltaik}

Für Solaranlagen werden die Ausschreibungen fortentwickelt und getrennte Ausschreibungen für große Dachanlagen und Freiflächenanlagen eingeführt. Die Einführung der Ausschreibungen für Dachanlagen beginnt im Jahr 2021 mit 200 MW für Dachanlagen größer $500 \mathrm{~kW}$. $\mathrm{Ab}$ dem Jahr 2023 wird das Marktvolumen unter Einbeziehung von Anlagen ab $300 \mathrm{~kW}$ und die Ausschreibungsmenge auf $400 \mathrm{MW}$ pro Jahr erhöht. Ab dem Jahr 2025 sollen Dachanlagen größer $100 \mathrm{~kW}$ an den Ausschreibungen teilnehmen. Das Ausschreibungsvolumen für Freiflächenanlagen bewegt sich kontinuierlich in einem Korridor zwischen 1,9 GW und 1,6 GW pro Jahr. Darüber hinaus ist bei Freiflächenanlagen mit einem wachsenden Anteil von Anlagen zu rechnen, die marktgetrieben realisiert werden, also außerhalb der Ausschreibungen. ${ }^{43}$ In der folgenden Abbildung ist die Ausschreibungsmenge und der prognostizierte Zubau außerhalb der Ausschreibung nach dem RefEnt EEG 2021 dargestellt.

Kritische Betrachtung: Für die PV sind in den Jahren 2021 bis 2028 jeweils Ausschreibungsmenge zwischen 1,9 und
2,8 GW vorgesehen. Die Mengen erhöhen sich ab 2025 stetig. Fraglich ist jedoch, ob angesichts der derzeitigen Flaute beim Ausbau der Windkraft, die insgesamt ausgeschriebenen Mengen reichen werden, um einen Erneuerbaren-Anteil von $65 \%$ bis $2030 \mathrm{zu}$ realisieren. Derzeit werden wegen Akzeptanzproblemen kaum neue Windparks in Deutschland realisiert und die Ausschreibungen sind regelmäßig unterzeichnet. ${ }^{44}$ Den Zubau der PV weiter zu beschränken, wie dies im EEG 2017 der Fall ist, wird von vielen Branchenvertretern bemängelt. Jede PVAusschreibungsrunde ist überzeichnet und die technologieübergreifende Ausschreibung mit der Windkraft wird von der PV dominiert. Ein PV-Ausbau mit einem hohen Volumen kann mit Freilandanlagen erreicht werden, die gut geplant Zusatzvorteile in der Fläche realisieren können. Dazu zählen beispielsweise Konzepte mit einer erhöhten Biodiversität. ${ }^{45}$

Des Weiteren wird eine Einführung separater Ausschreibungen für Dachanlagen von den meisten Branchenvertretern befürwortet. Zurzeit müssen Dachprojekte laut geltender Gesetzeslage mit Freiflächenanlagen in den Ausschreibungen ab $750 \mathrm{~kW}$ direkt konkurrieren und haben zumeist wenig Chancen. Für 2021 und 2022 plant die Regierung nun, jeweils $200 \mathrm{MW}$ für Dachanlagen separat auszuschreiben. Das Volumen wird dann in den Folgejahren noch deutlich steigen. Im Jahr 2028 soll es 1,2 GW erreichen. Mehrere Branchenvertreter weisen jedoch darauf hin, dass mit diesem geringen Ausschreibungsvolumen, gerade in den ersten Jahren, der Markt für große Dachanlagen deutlich ausgebremst wird. Nach den Zahlen der Bundesnetzagentur sind allein im vergangenen Jahr 1,12 GW an PV-Anlagen mit 500 bis $750 \mathrm{~kW}$ Leistung neu gemeldet worden. Zudem soll, wie bei den Freiflächenanlagen auch, bei den Dachanlagen, die Zuschläge über die Auktionen erhalten, kein Eigenverbrauch zulässig sein. ${ }^{46}$

\subsection{Fehlende Verbesserungen}

\subsubsection{Photovoltaikpflicht für Neubauten}

Einige Branchenvertreter fordern, dass PV-Anlagen bei Neubauten stärker eingebracht werden sollten. Bei jährlich rund 80.000 neuen Einfamilienhäusern ergibt sich ein

39) Bundesverband Erneuerbare Energie, Stellungnahme des Bundesverbands Erneuerbare Energie zum Entwurf eines Gesetzes zur Änderung des Erneuerbare-Energien-Gesetzes, 2020, S. 24.

40) Bundesverband der Energie- und Wasserwirtschaft, Stellungnahme zum Referentenentwurf für ein Gesetz zur Änderung des EEG, 2020, S. 67.

41) Bundesverband Erneuerbare Energie, Stellungnahme des Bundesverbands Erneuerbare Energie zum Entwurf eines Gesetzes zur Änderung des Erneuerbare-Energien-Gesetzes, 2020, S. 24

42) Deutsche Gesellschaft für Sonnenenergie, Referentenentwurf Änderung des Erneuerbare-Energien-Gesetzes und weiterer energierechtlicher Vorschriften, Stand 26.9.2020, abrufbar unter https://www.dgs.de/index.php?id=4341\&type=0, S. 1 .

43) Bundesministerium für Wirtschaft und Energie, Referentenentwurf - Entwurf eines Gesetzes zur Änderung des ErneuerbareEnergien-Gesetzes und weiterer energierechtlicher Vorschriften, 2020 , S. 83.

44) Enkhardt, EEG-Entwurf enthält Anschlussregelung für PostEEG-Anlagen und Ausschreibungen für PV-Dachanlagen, Stand 26.9.2020, abrufbar unter https://www.pv-magazine.de/2020/ 09/01/eeg-entwurf-enthaelt-anschlussregelung-fuer-post-eeganlagen-und-ausschreibungen-fuer-pv-dachanlagen/, S. 1.

45) Bundesverband Neue Energiewirtschaft, Stellungnahme zum Entwurf eine Gesetzes zur Änderung des Erneuerbare-Energien-Gesetzes, 2020, S. 14.

46) Enkhardt, EEG-Entwurf enthält Anschlussregelung für PostEEG-Anlagen und Ausschreibungen für PV-Dachanlagen, Stand 26.9.2020, abrufbar unter https://www.pv-magazine.de/ 2020/09/01/eeg-entwurf-enthaelt-anschlussregelung-fuer-posteeg-anlagen-und-ausschreibungen-fuer-pv-dachanlagen/, S. 1. 
Potenzial von zusätzlich 600 Mio. Kilowattstunden Solarstrom jährlich. ${ }^{47}$

\subsubsection{Erweiterung der Flächenkulisse}

Schwimmende PV-Anlagen, wie sie gegenwärtig z.B. verstärkt in den Niederlanden zur Anwendung kommen, würde gerade ehemaligen Kohlerevieren eine interessante Perspektive bieten. Dazu bietet die Kombination von Landwirtschaft und PV-Anlagen (Agri-PV) positive Synergien und vermeidet Flächenkonkurrenzen. Die Erzeugungskosten von Solarstrom aus beiden PV-Technologien liegen konstruktionsbedingt etwas über denen herkömmlicher Solarparks. Im Wettbewerb mit diesen um Zuschläge im Rahmen der regulären PV-Auktionen wären sie weiter chancenlos. Für innovative PV-Marktsegmente schwimmende PV und Agri-PV sollte ein Ausschreibungssegment mit einem über fünf Jahre kontinuierlich aufwachenden Auktionsvolumen eingeführt werden. In dem Förderprogramm sollte dabei sichergestellt werden, dass jeder einzelnen Technologie eine Mindestmenge zugeschlagen wird, um ein Markthochlauf in den unterschiedlichen neuen Technologien sicherzustellen. ${ }^{48}$

\section{Zusammenfassung und Ausblick}

Das Bundesministerium für Wirtschaft und Energie hat am 14. 9. 2020 den Gesetzesentwurf zur Novellierung des EEG veröffentlicht und den Ländern und Branchenverbänden zur Konsultation zukommen lassen. Mit dem Gesetzesentwurf soll das geltende EEG 2017 durch ein grundlegend novelliertes EEG ersetzt werden, das zum 1.1.2021 in Kraft treten soll. Die vorliegende Ausarbeitung hat die bedeutendsten Änderungen für die Stromerzeugung aus PV identifiziert und analysiert. Folgend werden die wichtigsten Ergebnisse zusammengefasst.

Die Ausbaupfade und Ausschreibungsmengen im RefEnt basieren auf den Annahmen des Klimaschutzprogramms, welche einen sinkenden Stromverbrauch auf rund 580 TWh zur Berechnung des 65\%-Ziels im Jahr 2030 prognostizieren. Jedoch wird, aus Sicht von zahlreichen Marktforschern, der Stromverbrauch auf mindestens $740((1 / 6))$ TWh steigen. Dies sollte sich auch in den im Rahmen einer Novellierung des EEG angepassten Ausbaupfaden und Ausschreibungsmengen widerspiegeln.

Eine weitere technologieübergreifende Änderung ist die Änderung der Sechs-Stunden-Regel. Die bisherige SechsStunden-Regel gemäß $₫ 51$ Abs. 1 EEG 2017 gefährdet die Wirtschaftlichkeit und Planbarkeit von Erneuerbaren-Energien-Anlagen. Der RefEnt schlägt vor, bei einer Zeitspanne von 15 Minuten negativer Preisen schon keine Marktprämie mehr zu gewähren. Dies verschärft die Situation noch einmal zusätzlich. Viele Branchenvertreter, darunter der Bundesverband für Erneuerbare-Energien, fordern deshalb eine Abschaffung des $₫ 51$ im EEG.

Ferner strebt die Bundesregierung eine Weiterentwicklung der Digitalisierung im Energiesektor an. Lösungen müssen aber den Anforderungen der Marktakteure genügen. Den Pflichteinbau intelligenter Messsysteme bei Erneuerbare-Energien-Anlagen ab einer Leistung von $1 \mathrm{~kW}$ vorzusehen, ist nach Meinung vieler Branchenvertretender überzogen. Die zusätzlich erforderliche Messtechnik darf die Erlöse der Anlagen nicht aufzehren.

Eine weitere maßgebende Änderung betrifft den Mieterstrom. Die meisten Branchenverbände begrüßen die
Einführung eines gestaffelten Mieterstromzuschlags gemäß \48a RefEnt EEG 2021. Allerdings sollte der Mieterstromzuschlag auf 3,5 bis $4 \mathrm{ct} / \mathrm{kWh}$ festgelegt werden. Mit dieser Maßnahme kann der administrative Aufwand für eine begrenzte Zeit ausgeglichen werden.

Photovoltaik spezifisch sieht der RefEnt die Einführung eines Ausschreibungssystems für Gebäude-PV-Anlagen vor. Die Ausschreibungsgrenze soll dabei von $750 \mathrm{kWp}$ schrittweise gesenkt werden. Eine Absenkung wird von Branchenverbänden als kritisch betrachtet. Sie befürchten, dass der Ausbau in einem der wichtigsten PV-Marktsegmente dadurch ausgebremst wird. Möglichkeiten für mittelständische Investoren in energiewendedienliche Anwendungen zu investieren werden dadurch erheblich erschwert.

Dagegen treffen die erhöhte Gebotsgröße von $20 \mathrm{MW}$ und ausgeweitete Flächenkulisse an Verkehrswegen auf 220 Meter auf durchweg positive Reaktionen. Lediglich die Regelung in Bezug auf die realen Flurstücke sollte flexibler ausgelegt werden.

Ein weiterer wichtiger Punkt ist die Regelung der weiterführenden Förderung nach Ablauf der Vergütungsperiode. Der Entwurf sieht vor, Anlagen bis höchstens $100 \mathrm{~kW}$ eine zweite Anschlussperspektive zu bieten. Der Netzbetreiber ist weiterhin dazu verpflichtet die Abnahme und Vermarktung des erzeugten Stroms zu übernehmen.

Letztlich ist festzuhalten, dass die Ausschreibungshöhe für PV-Dachanlagen zu niedrig angesetzt ist. Mehrere Branchenvertreter weisen darauf hin, dass mit diesem geringen Ausschreibungsvolumen, gerade in den ersten Jahren, der Markt für große Dachanlagen deutlich ausgebremst wird.

Das Bundeskabinett hat den Gesetzentwurf am 23.9.2020 verabschiedet. Das EEG 2021 wird nun in einem nächsten Schritt im parlamentarischen Verfahren im Bundestag und Bundesrat beraten. Ziel ist der Abschluss des Gesetzgebungsverfahrens noch in diesem Jahr. Es bleibt mit Spannung abzuwarten, welche Verbesserungsvorschläge der zahlreichen Stellungnahmen der Branchenverbände aufgegriffen werden.

Open Access. Dieser Artikel wird unter der Creative Commons Namensnennung 4.0 International Lizenz veröffentlicht, welche die Nutzung, Vervielfältigung, Bearbeitung, Verbreitung und Wiedergabe in jeglichem Medium und Format erlaubt, sofern Sie den/die ursprünglichen Autor(en) und die Quelle ordnungsgemäß nennen, einen Link zur Creative Commons Lizenz beifügen und angeben, ob Änderungen vorgenommen wurden.

Die in diesem Artikel enthaltenen Bilder und sonstiges Drittmaterial unterliegen ebenfalls der genannten Creative Commons Lizenz, sofern sich aus der Abbildungslegende nichts anderes ergibt. Sofern das betreffende Material nicht unter der genannten Creative Commons Lizenz steht und die betreffende Handlung nicht nach gesetzlichen Vorschriften erlaubt ist, ist für die oben aufgeführten Weiterverwendungen des Materials die Einwilligung des jeweiligen Rechteinhabers einzuholen.

Weitere Details zur Lizenz entnehmen Sie bitte der Lizenzinformation auf http://creativecommons.org/licenses/by/4.0/deed.de.

Open Access funding enabled and organized by Projekt DEAL.

47) Enkhardt, Kabinett beschließt EEG-Entwurf, Stand 26.9.2020, abrufbar unter https://www.pv-magazine.de/2020/09/23/kabinett-beschliesst-eeg-entwurf/, S. 1.

48) Bundesverband Solarwirtschaft, Stellungnahme EEG-Novelle 2021, 2020, S. 22. 